JENA-FKS-94-2

\title{
PHASE-LOCKING IN STRONGLY COUPLED SQUID CELLS
}

\author{
M. Basler, W. Krech ${ }^{\dagger}$ and K. Yu. Platov $\$ \S$ \\ Friedrich-Schiller-Universitt Jena \\ Institut fr Festkrperphysik \\ 07743 Jena \\ Max-Wien-Platz 1
}

October 12, 2018

\begin{abstract}
Within the RSJ model we performed an analytical and numerical investigation of SQUID cells consisting of two Josephson junctions shunted by an extremely small inductance leading to strong coupling of the elements. Contrary to the well-known behavior of cells shunted by a high inductance voltage phases of the junctions are locked with very small phase difference for almost all values of external flux. Only for external flux in the vicinity of half a flux quantum phase difference rises rapidly to $\pi$.
\end{abstract}

Submitted to Physics Letters A

Typeset with $\mathrm{LT}_{\mathrm{E}} \mathrm{X}$

*pmb@rz.uni-jena.de

†owk@rz.uni-jena.de

†okp@rz.uni-jena.de

§Permanent address: Laboratory of Cryoelectronics, Physics Department, Moscow State University, Moscow 119899 Russia 
There has been growing interest in the development of complex Josephson junction arrays during last years being caused by possible applications in the field of satellite communication systems, astronomical observations or construction of supercomputer chips [1-4]. Despite some promising experimental results with 2D arrays [5-9] detailed theoretical studies are still missing. In addition, nearly all theoretical investigations on coupled Josephson junctions in general are based on weakly coupled elements. Otherwise, present day and near future technology will allow construction of arrays with elements strongly coupled by inductances around $3 \mathrm{pH}$ and smaller.

Thus before attacking more complex problems it is useful to perform theoretical studies of very simple arrays of strongly coupled Josephson junctions. One of the simplest arrays at all is a completely superconducting SQUID cell consisting of two identical Josephson junctions with parallel current support inductively shunted by a very small ( $L \ll \phi_{0} / 2 \pi I_{C} ; \quad \phi_{0}$ : flux quantum, $I_{C}$ : critical current) inductance. Phase-locking in this model has been studied thoroughly in the weak-coupling limit several years ago [1, 3, [1], but any detailed theoretical investigations on strong inductive coupling seem to be missing so far. The results of our work show that the behavior of strongly coupled Josephson junctions, especially under the influence of external magnetic fields $\phi$, is qualitatively different from what has been known for weakly coupled elements.

The circuit to be considered here is shown in Fig. 1.

Both junctions, to be considered as being identical and having negligible capacitance, are described within the RSJ model. The behavior for weak coupling $\left(L \gg \phi_{0} / 2 \pi I_{C}\right)$ can be summarized as follows: (i) In any case there exists a stable phase-locking regime. (ii) Without an external magnetic field the difference between Josephson phases on both junctions vanishes in the mean. In this case there is no stationary current flow through the shunt. (iii) Any external flux $\phi$ leads to a non-vanishing difference of Josephson phases of the elements causing a phase difference $\delta$ between voltages. The mean value of this voltage phase difference $\delta$ can be determined by the so-called reduced equation $[1,10]$

$$
\begin{gathered}
\delta-\frac{1}{i_{0}\left(i_{0}+\sqrt{i_{0}^{2}-1}\right)} \sin \delta=\varphi, \\
i_{0}=I_{0} / I_{C}, \quad \varphi=2 \pi \phi / \phi_{0} .
\end{gathered}
$$

Thus, for usual operation regimes with $i_{0} \approx 1.5$ the $\delta-\varphi$ dependence is nearly linear (cf. Fig. 2).

This may cause problems in larger arrays; if parasitic magnetic fluxes cannot be suppressed radiation output will be small because of cancellations between contributions of different cells.

Here we consider the behavior for strong inductive coupling, $L \ll \phi_{0} / 2 \pi I_{C}$. For vanishing McCumber parameter $\beta_{C}$ the circuit can be described by the RSJ equations

$$
\begin{gathered}
\dot{\phi}_{1}+\sin \phi_{1}=i-l^{-1}\left(\phi_{1}-\phi_{2}+\varphi\right), \\
\dot{\phi}_{2}+\sin \phi_{2}=i+l^{-1}\left(\phi_{1}-\phi_{2}+\varphi\right)
\end{gathered}
$$


where

$$
l=2 \pi I_{C} L / \phi_{0}<<1
$$

is the dimensionless inductive coupling and the dot denotes the derivative with respect to the normalized time

$$
s=\frac{2 e}{\hbar} R_{N} I_{C} t
$$

For obtaining approximate solutions in the strong coupling limit one introduces new variables

$$
\Delta=\phi_{2}-\phi_{1} \quad \text { and } \quad \Sigma=\phi_{2}+\phi_{1}
$$

and applies an expansion for small $l$

$$
\begin{aligned}
\Delta & =\Delta_{0}+l \Delta_{1}+\mathcal{O}\left(l^{2}\right), \\
\Sigma & =\Sigma_{0}+l \Sigma_{1}+\mathcal{O}\left(l^{2}\right) .
\end{aligned}
$$

This analytical approach was complemented by a numerical investigation exploiting the Personal Supercomputer Circuit ANalyzer program PSCAN [11, 12] We will not go into the details of calculation here, but concentrate on physical results.

For vanishing external flux the elements behave in the same way as two weakly coupled elements; phase shift between voltages vanishes and elements oscillate oppositely with voltages

$$
\begin{array}{r}
v_{1}=v_{2}=\frac{\zeta_{0}^{2}}{i_{0}+\cos \left(\zeta_{0} s-\delta_{0}\right)}, \\
\zeta_{0}=\sqrt{i_{0}^{2}-1}, \quad \delta_{0}=\text { const }, \quad v_{k}=\frac{V_{k}}{I_{C} R_{N}} .
\end{array}
$$

The behavior is quite plausible, because in this case there is no current flow through the shunt.

If the external flux does not vanish, the behavior is more complicated. To first order in $l$, time dependent voltage is obtained as follows:

$$
\begin{aligned}
& v_{1 / 2}=\frac{\bar{\zeta}_{0}^{2}}{i_{0}+\cos (\varphi / 2) \cos \bar{\zeta}_{0} s}+l \frac{\bar{\zeta}_{0} \sin (\varphi / 2)}{2\left(i_{0}+\cos (\varphi / 2) \cos \bar{\zeta}_{0} s\right)^{2}} \times \\
& {\left[\sin (\varphi / 2) \sin \bar{\zeta}_{0} s\left(i_{0}+\cos (\varphi / 2)+\frac{\bar{\zeta}_{0}^{2}}{\cos (\varphi / 2)} \ln \frac{i_{0}+\cos (\varphi / 2) \cos \bar{\zeta}_{0} s}{i_{0}+\cos (\varphi / 2)}\right)\right.} \\
& \left.\mp \bar{\zeta}_{0}\left(\cos (\varphi / 2)+i_{0} \cos \bar{\zeta}_{0} s\right)\right]
\end{aligned}
$$

where

$$
\bar{\zeta}_{0}=\sqrt{i_{0}^{2}-\cos ^{2}(\varphi / 2)}
$$

This solution shows several specific features. (i) As in the case of weak coupling there exists a solution exhibiting phase locking for all values of the external field. (ii) For extremely strong coupling $l \rightarrow 0$ both elements behave similar to free junctions, but with 
the current $i_{0}$ substituted by a kind of "effective current" depending on external flux, $i_{0} / \cos (\varphi / 2)$. (ii) There is a phase shift between voltage phases being caused by the last term of Eq. (12). However, contrary to the weak coupling case, this phase shift is very small over a wide range of external flux and only in the vicinity of the value $\varphi=\pi$ the phase shift jumps to the value $\delta=\pi$. Within the range of validity of our perturbation theory it is possible to derive an explicit analytical expression for the phase shift $\delta$ :

$$
\cos \delta=\frac{\left(b^{2}+a_{1} a_{2}\right)}{\sqrt{b^{4}+b^{2}\left(a_{1}^{2}+a_{2}^{2}\right)+a_{1}^{2} a_{2}^{2}}}
$$

where $a_{1}, a_{2}$ and $b$ are the leading Fourier coefficients of the voltages (12)

$$
\begin{aligned}
a_{1 / 2} & =-2 \sqrt{\frac{i_{0}-\bar{\zeta}_{0}}{i_{0}+\bar{\zeta}_{0}}} \mp l \sin (\varphi / 2) \frac{\bar{\zeta}_{0}}{i_{0}+\bar{\zeta}_{0}}, \\
b & =l \sin ^{2}(\varphi / 2)\left(\frac{i_{0}-\bar{\zeta}_{0}}{i_{0} \bar{\zeta}_{0}}+\frac{1}{\bar{\zeta}_{0}} \sqrt{\frac{i_{0}-\bar{\zeta}_{0}}{i_{0}+\bar{\zeta}_{0}}}+\frac{\bar{\zeta}_{0}^{2}}{4 i_{0}^{2}\left(i_{0}+\bar{\zeta}_{0}\right)} \sqrt{\frac{i_{0}-\bar{\zeta}_{0}}{i_{0}+\bar{\zeta}_{0}}}\right) .
\end{aligned}
$$

Fig. 3 shows a plot of phase shift between junctions against external field.

This can be compared with results from numerical simulation (including a small shunt capacitance $\beta_{C}=0.01$; cf. Fig. 4).

The limiting case $\varphi=\pi$ is of special interest:

$$
\begin{aligned}
& v_{1}=i_{0}-\frac{l i_{0}}{2} \sin \left(i_{0} s-\delta_{0}\right)+\frac{l}{4} \sin 2\left(i_{0} s-\delta_{0}\right), \\
& v_{2}=i_{0}+\frac{l i_{0}}{2} \sin \left(i_{0} s-\delta_{0}\right)+\frac{l}{4} \sin 2\left(i_{0} s-\delta_{0}\right) .
\end{aligned}
$$

This clearly indicates the phase difference between both voltages to first order being equal to $\delta=\pi$.

Comparison of Figs. 3 and 4 shows that even for $l \approx 1$ where our analytical approximation is not longer valid results are not far from that of numerical simulation. Both figures show that already in this case the behavior is qualitatively different from what has been known for weakly coupled elements (dotted lines in Figs. 3 and 4).

Within the interval $0 \leq \varphi<\pi$ Eq. (14) can be rewritten as

$$
\delta(\varphi)=\arctan \left(a_{2} / b\right)-\arctan \left(a_{1} / b\right) .
$$

This shows, that for $l \ll 1$, but finite, the solution can be approximated by a step function

$$
\delta \approx \pi \theta\left(\varphi-\varphi^{*}\right)
$$

with the step at

$$
\varphi^{*}=\pi-i_{0} l
$$

(cf. Fig. 5). 
Clearly $\varphi^{*} \rightarrow 0$ for $l \rightarrow 0$. This approximation might be useful considering more complicated arrays.

To conclude, our investigation of two strongly coupled Josephson junctions has shown, that for every value of the external field there exists a regime showing phase-locking between voltage phases. Contrary to the case of weak coupling via large inductances, phase shift between voltages is negligibly small for a wide range of external flux for small inductive shunts, but shows a narrow peak centered around the value $\varphi=\pi$. If larger arrays will show a similar behavior, which has to be investigated in more detail, this would make them nearly insensible to parasitic external flux.

\section{References}

[1] A.K. Jain, K.K. Likharev, J.E. Lukens, J.E.Sauvageau, Phys. Rep. 109 (1984) 310.

[2] J.B. Hansen, P.E. Lindelof, Rev. Mod. Phys. 56 (1984) 431.

[3] K.K. Likharev, Dynamics of Josephson Junctions and Circuits (Gordon and Breach, Philadelphia, 1991).

[4] S.P. Benz, C.J. Burroughs, Supercond. Sci. Technol. 4 (1991) 561.

[5] T.D. Clark, Phys. Lett. A 27 (1968) 585.

[6] T.D. Clark, Phys. Rev. B 8 (1973) 137.

[7] S.P. Benz, C.J. Burroughs, Appl. Phys. Lett. 58 (1991) 2162.

[8] B. Bi, S. Han, J.E. Lukens, K. Wan, IEEE Trans. Appl. Supercond. 3 (1993) 2303.

[9] P.A.A. Booi, S.P. Benz, IEEE Trans. Appl. Supercond. 3 (1993) 2493.

[10] W. Krech, Wiss. Z. Jena, Math.-Nat. R. 32 (1983) 19.

[11] A.A. Odintsov, V.K. Semenov, A.B. Zorin, IEEE Trans. Magn 23 (1987) 763.

[12] S.V. Polonsky, V.K. Semenov, P.N. Shevchenko, Proc. of ISEC'91, Scotland, (1991) 160. 


\section{Figure Captions}

Fig 1. Circuit of two Josephson junctions with superconducting inductive SQUID coupling.

Fig 2. Mean voltage phase shift $\delta$ against normalized external field $\varphi$ from analytical approximation for weak inductive coupling $\left(i_{0}=1.5\right)$.

Fig 3. Mean phase shift $\delta$ against normalized external filed $\varphi$ for strong inductive coupling

$l=0.1$ and medium inductive coupling $l=1.0$ obtained from analytical approximation (14) $\left(i_{0}=1.5\right)$.

Fig 4. Mean phase shift $\delta$ against normalized external filed $\varphi$ for strong inductive coupling $l=0.1$ and medium inductive coupling $l=1.0$ obtained by numerical simulation $\left(i_{0}=1.5\right)$.

Fig 5. Mean phase shift $\delta$ against normalized external field $\varphi$ for extremely strong coupling $(0.001 \leq l \leq 0.1)$ obtained from analytical approximation (14) $\left(i_{0}=1.5\right)$. 
Fig.1

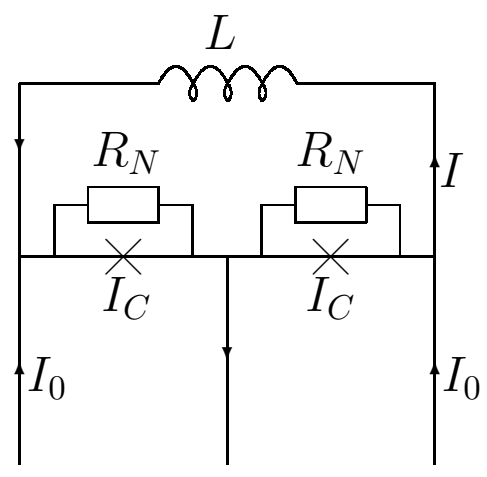


Fig.2

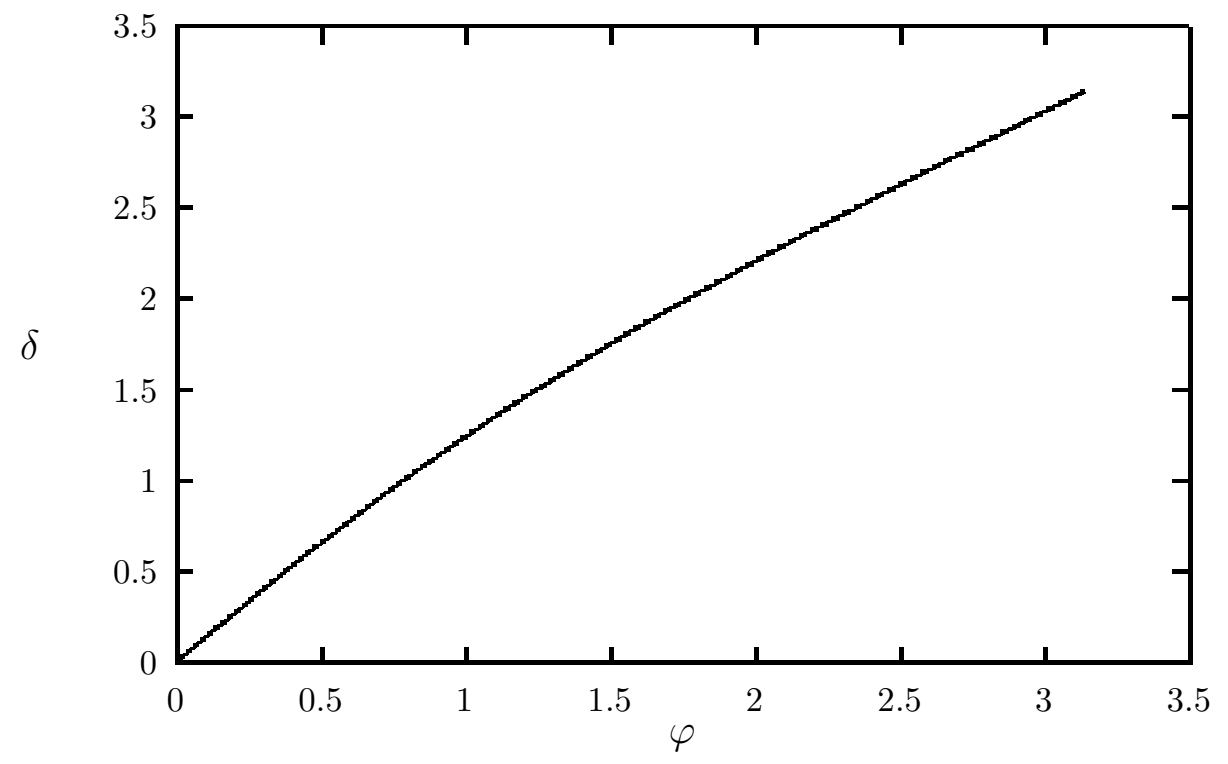


Fig.3

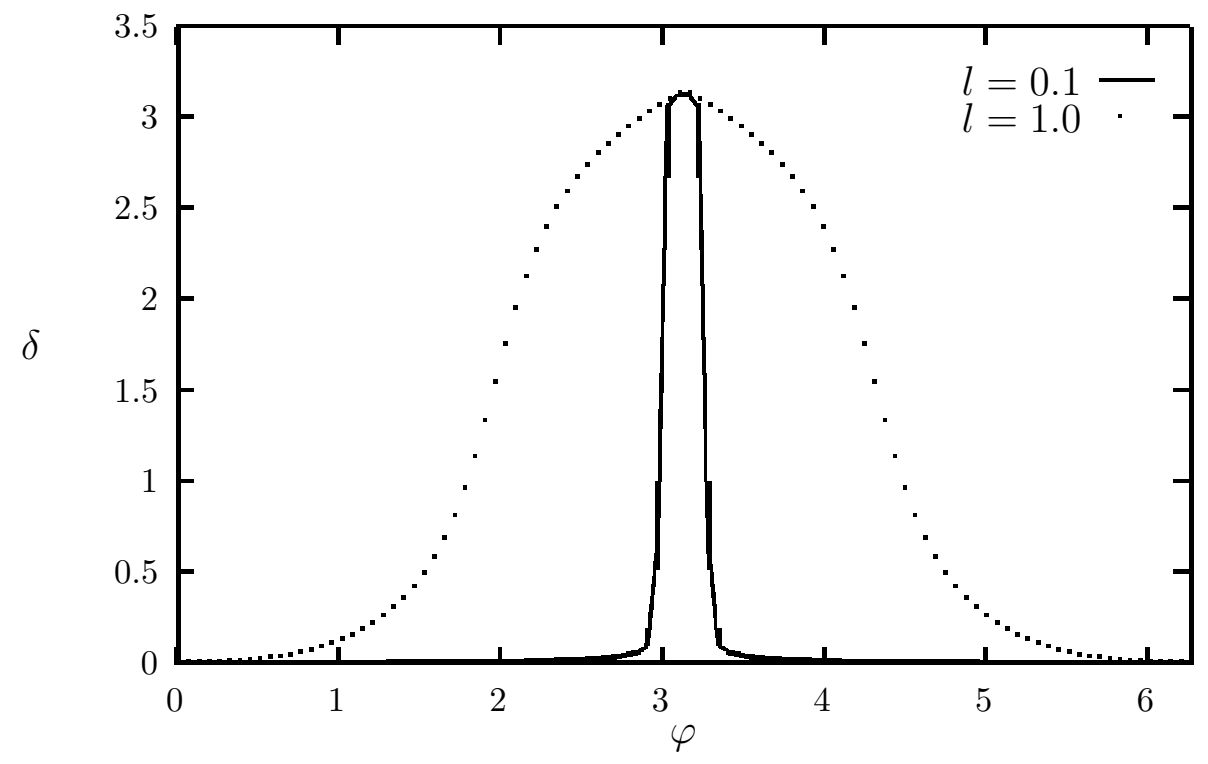


Fig.4

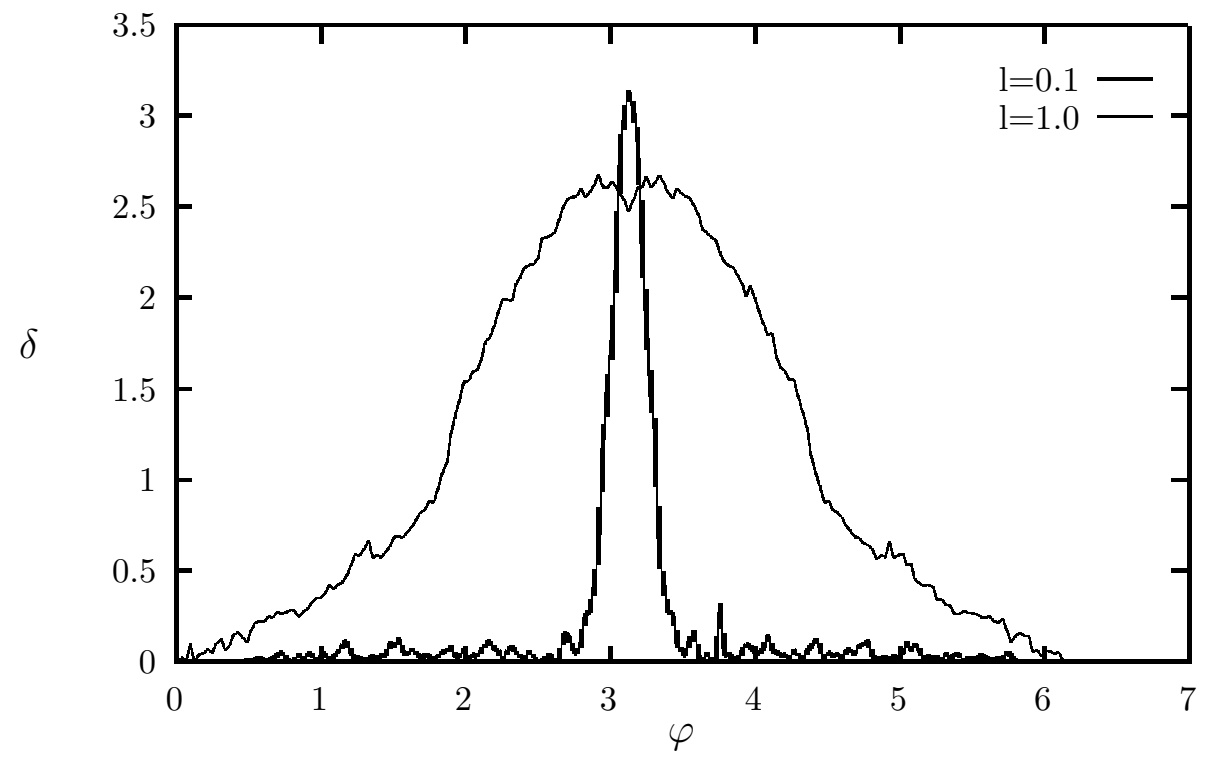


Fig.5

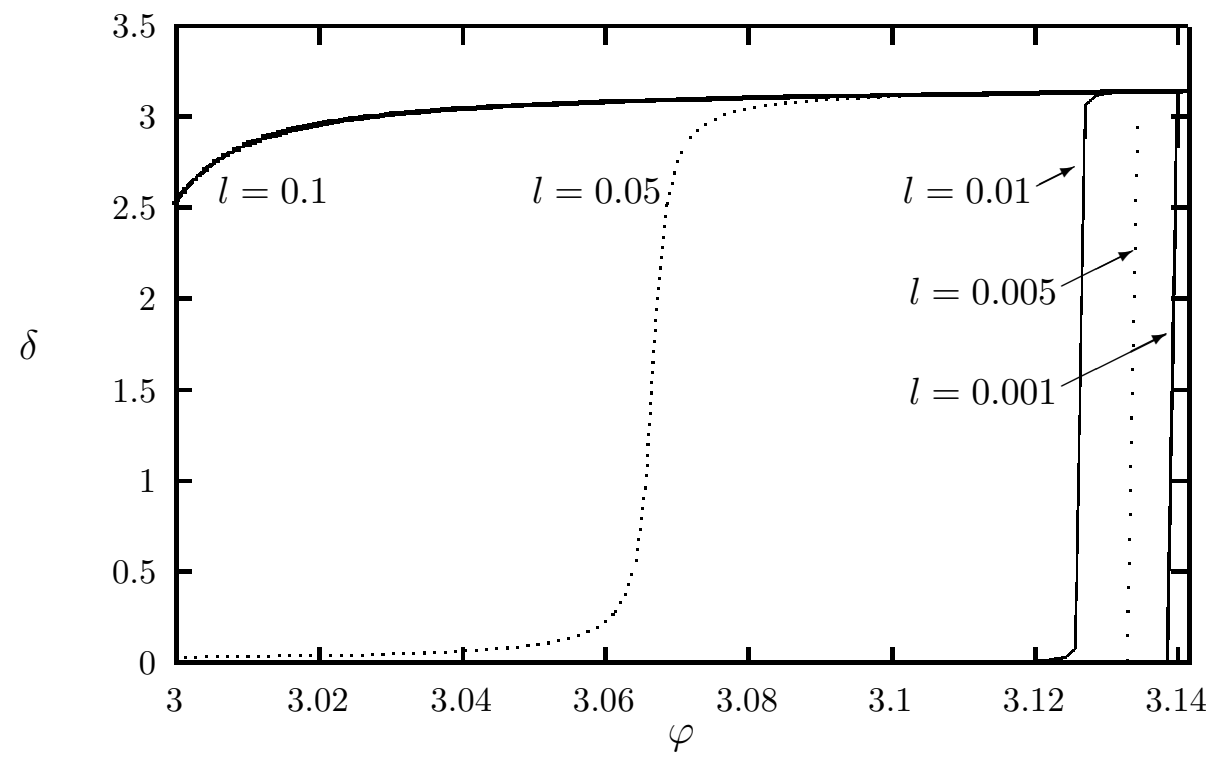

